\title{
Albumin Paclitaxel Plus Platinum With or Without Pembrolizumab for Metastatic Primary Pulmonary Lymphoepithelioma-like Carcinoma: a Retrospective Multicenter Study
}

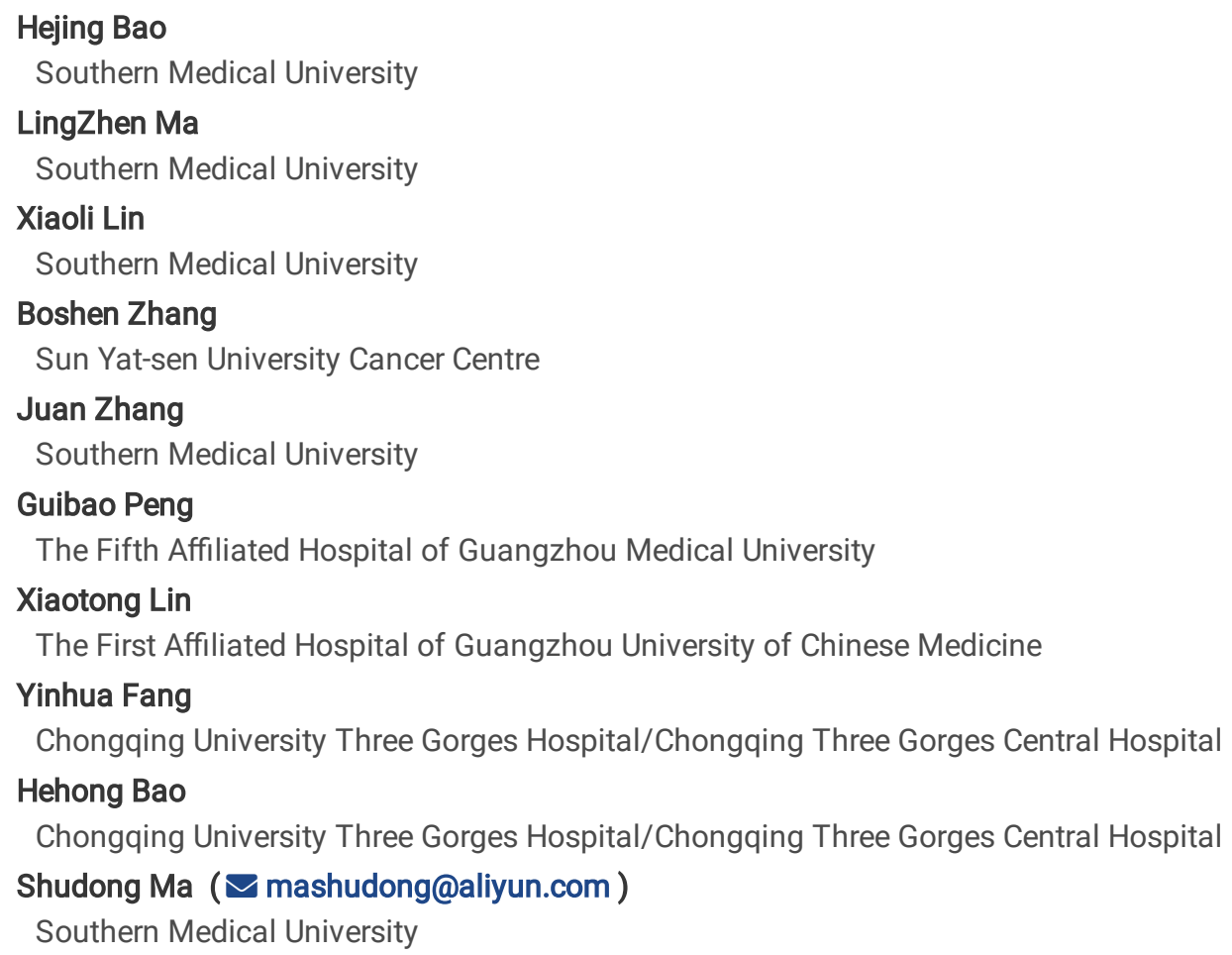

\section{Research Article}

Keywords: primary pulmonary lymphoepithelioma-like carcinoma, immune checkpoint inhibitor, efficacy and safety, retrospective study, FBXW7 mutation

Posted Date: October 21st, 2021

DOI: https://doi.org/10.21203/rs.3.rs-966201/v1

License: (c) (i) This work is licensed under a Creative Commons Attribution 4.0 International License. Read Full License 


\section{Abstract \\ Objectives}

Primary pulmonary lymphoepithelioma-like carcinoma (PPLELC) is a rare subtype of primary non-small cell lung cancer (NSCLC), and the first-line therapy for metastatic PPLELC patients remains controversial. The purpose of this study was to investigate the efficacy and safety of immune checkpoint inhibitors(ICls) combined with chemotherapy(CT) compared with traditional chemotherapy in these patients.

\section{Methods}

A total of 168 patients with metastatic PPLELC came from six grade A hospitals from August 2018 to August 2020 were selected. 17 patients were enrolled in the ICl group and received $200 \mathrm{mg}$ of Pembrolizumab plus albumin paclitaxel and carboplatin every 3 weeks. 34 patients with chemotherapy alone were assigned to the CT group and received albumin-paclitaxel combined with carboplatin every 3 weeks.

\section{Results}

As of June 1, 2021, the median PFS was 14.9 months for ICl group and 6.4 months for CT group [Hazard Ratio (HR), 0.29; 95\% confidence interval $(\mathrm{Cl}), 0.15-0.55 ; \mathrm{P}<0.05$ ]. ORR was $64.7 \%$ in ICl group and $35.3 \%$ in $\mathrm{CT}$ group [HR, $0.65 ; 95 \% \mathrm{Cl}, 0.39-0.90 ; \mathrm{P}=0.047$ ]. The median OS of ICl group was not reached, while that of CT group was 13 months. In the ICl group, there were 8 cases (47.1\%) of grade 3 treatmentrelated adverse reactions and 5 cases $(29.4 \%)$ of grade 4 treatment-related adverse reactions. In the CT group, there were 9 cases $(26.5 \%)$ of grade 3 treatment-related adverse reactions and 8 cases $(23.5 \%)$ grade 4 treatment-related adverse reactions. FBXW7 mutation were negatively and TP53 mutation, MRE11A p.V198S mutation, PTEN p.T319FS mutation were positively correlated with the efficacy of immunotherapy.

\section{Conclusions}

In patients with metastatic PPLELC, the efficacy of immune checkpoint inhibitors combined with chemotherapy was significantly better than that of chemotherapy alone, and adverse reactions were acceptable.

\section{Introduction}

Lymphoepitheliomatoid carcinoma is a rare epithelial neoplasm, mostly originates from the nasopharynx, but also occurs in the foregut of origin, also has been reported occur in lung, stomach, thymus, liver, cervix, salivary glands and bladder, and is rarely seen in the ovary ${ }^{12}$. Primary pulmonary lymphoepithelioma-like carcinoma is a rare subtype of primary non-small cell lung cancer that histologically resembles undifferentiated nasopharyngeal carcinoma (NPC) ${ }^{3}$, its incidence in all cases of non-small cell lung cancer is about $0.7 \%{ }^{24}$. It was previously classified as a variant of large cell carcinoma ${ }^{5}$, then reclassified as other and unclassified cancers in the world Health Organization (WHO) Classification of Lung tumors in $2015^{6}$. First described by Begin et al.in 1987 ${ }^{7}$, PPLELC has been considered to be closely associated with epstein-Barr virus (EBV) infection ${ }^{8}$. It has the unique morphological characteristics of undifferentiated carcinoma with a typical syncytial growth pattern with large vesicle nuclei with prominent nucleoli and abundant lymphocyte infiltration 7 . Tumors are usually positive for CK5/6, EMA, P63, and P40, suggesting a squamous cell lineage ${ }^{9,10}$. The presence of EBV in the nuclei of tumor cells is necessary for diagnosis and detection of EBV-encoded RNA(EBER) by in situ hybridization is essential ${ }^{11}$. Although histologically similar to undifferentiated nasopharyngeal carcinoma with lymphocytic infiltration, nonclassical histological morphology of lung lymphoepitheliomatoid carcinoma has been observed, including EBV negative ${ }^{12}$ and heterogeneity in the degree of lymphocytic infiltration ${ }^{13}$.

About 1,600 cases have been reported worldwide in the past 33 years since the discovery ${ }^{4,14-18}$, mainly focused on the past five years, reporting mainly in Asia, especially Hong Kong, Taiwan, Guangdong and other regions. PPLELC usually affects never smokers, is genderneutral, and is younger than non-small cell lung cancer ${ }^{19}$. There are no obvious clinical manifestations of PPLELC. Cough, chest pain, hemoptysis and even slight fever are typical clinical manifestations of primary PPLELC patients ${ }^{20}$. CT scans often show an isolated nodule $^{21}$. As with all neoplastic lesions, the diagnosis of primary PPLELC is entirely dependent on histopathological examination, but the morphologic similarity of primary PPLELC to poorly differentiated squamous cell carcinoma adds to the difficulty of diagnosis. Similar to other types of lung cancer, the prognosis is good for most patients diagnosed early with PPLELC, with a median overall survival of

Page 2/16 
approximately 107 months and a 5-year survival of approximately $60 \%$, compared with a mean survival of 13 months for patients with nonlung lymphoepithelioid 22,23 .

The main treatment strategy for early disease is surgery ${ }^{19,24}$. Patients with distant metastases who cannot be treated surgically are often treated with multiple treatment modalities ${ }^{4,14,15,25-29}$. Several treatments have been reported in the literature, but the treatment of PPLELC is empirical due to its rarity. In addition, the use of targeted therapy and immunotherapy in PPLELC is limited by a lack of information on the molecular mechanisms underlying their tumorigenesis. Patients with PPLELC may not benefit from personalized, targeted therapies commonly used in NSCLC. At present, immunotherapy has become a promising treatment for NSCLC. However, for patients with metastatic PPLELC, there is limited and unconvincing evidence on the clinical effects of immune checkpoint inhibitors. Therefore, this study aims to investigate the efficacy of immune checkpoint inhibitors combination in patients with metastatic PPLELC.

\section{Methods}

\section{Patients}

Patients aged 18 years or older, stage IIIB/IV diagnosed with metastatic PPLELC according to the 2015 WHO histological classification criteria for lung tumors, and without allergenic EGFR or ALK mutations, were eligible for inclusion; The performance status score by Eastern Cooperative Oncology Group (ECOG) is 0 or 1(on a 5-point scale, higher scores indicate more severe disability); According to the Solid Tumor Response Assessment Criteria (RECIST), version 1.1 has at least one measurable lesion. Patients were excluded if they had symptomatic CNS metastases, autoimmune disease, symptomatic interstitial lung disease, systemic immunosuppression, prior treatment with T-cell costimulation or checkpoint targeted drugs. All patients underwent nasopharyngeal endoscopy to exclude metastatic lymphoepitheliomatoid carcinoma of the nasopharynx.

Follow-up information of patients was obtained through inpatient medical records, outpatient data and telephone follow-up. Follow-up survival was calculated from the first day of inclusion, and the follow-up deadline was June 1, 2021. This study was approved by the Ethics Review Board of Nanfang Hospital, Southern Medical University (Guangzhou, China). Informed consent was waived because the study was retrospective. This retrospective study collected 168 cases of PPLELC patients from Nanfang Hospital affiliated to Southern Medical University, Zhujiang Hospital affiliated to Southern Medical University, The First Affiliated Hospital of Guangzhou University of Traditional Chinese Medicine, Cancer Hospital of Sun Yat-sen University, Cancer Hospital affiliated to Guangzhou Medical University, Three Gorges Hospital affiliated to Chongqing University in China in August 2018 to August 2020, 51 cases were included in the study. (figure1)

\section{Experimental design and treatment}

In this real-world retrospective study, patients were divided into ICl group with first-line immunization combined with chemotherapy and CT group with first-line chemotherapy. First-line immunization combined with chemotherapy received 200 mg Pembrolizumab and albumin paclitaxel combined with carboplatin every 3 weeks; The first-line chemotherapy group received albumin paclitaxel every 3 weeks in combination with carboplatin. Treatment until radiographic progression or unacceptable toxicity.

\section{Assessment}

PD-L1 expression was assessed by PD-L1 IHC 22C3 pharmDx assay (Agilent) on formalin fixed tumor samples obtained from biopsy or excision biopsy or from tissue resected for metastatic disease. Expression was classified according to tumor proportion score (i.e., percentage of tumor cells with membranous PD-L1 staining). Pd-I1 expression was expressed by tumor proportion score, 0-1\% was negative, low expression score ranged from $1-49 \%$, and high expression score was $\geq 50 \%$. All slides were scored for PD-L1 membrane staining by two independent pathologists. Postoperative staging the latest 8th edition of primary tumor-node-metastasis (TNM) staging system for lung cancer was used for staging or clinical staging. Adverse events and laboratory abnormalities were graded according to the National Cancer Institute Standard for Common Terminology for Adverse Events, Version 4.0. The tumor response assessment program is conducted every two to four cycles. Responses were assessed according to RECIST version 1.1.

\section{Terminus}

The primary end points were progression-free survival (time from inclusion to disease progression or death from any cause, whichever came first), and the secondary end points were overall survival (time from randomization to death from any cause), response rate (percentage of patients with confirmed complete or partial response) and safety. Remission rates and duration were assessed by independent radiological assessment. Exploratory endpoints included the effect of genetic mutations on efficacy and patient outcomes.

\section{Statistical analysis}


Efficacy was assessed in the enrolled population and safety was assessed in the treated population. Kaplan-meier methods were used to estimate overall survival and progression-free survival. Data on patients who survived or were lost to follow-up were reviewed to see their overall survival at the time of their last survival. At the time of the last radiographic assessment, data from patients who had survived without disease progression or failed follow-up were examined to analyze progression-free survival. T test was used for measurement data (age) and Chi-square test (Cartesian test) was used for counting data. All statistical analyses were performed in IBM SPSS Statistics(Version 19.0, Armonk, NY, USA). When P value was less than 0.05 , the difference was considered statistically significant.

\section{Results}

The median age of patients was 54 years (46.5 to 57 years). There were 28 female patients (54.9\%) and 23 male patients (45.1\%). ECOG score was 0 in 31 patients (60.8\%) and 1 in 20 patients (39.2\%). 17 patients (33.3\%) were smokers, and 18 patients (15.7\%) had a family history. There were 34 patients (66.7\%) with tumor size $\geq 4 \mathrm{~cm}$, and 17 patients $(33.3 \%)$ with tumor size $<4 \mathrm{~cm}$. There were 20 cases $(39.2 \%)$ with single organ metastasis, and 31 cases (60.8\%) with multiple organ metastasis. The most common tumor site was the middle lobe of the right lung, with 17 cases (33.3\%), followed by 14 cases (27.5\%) in the left lower lobe, 10 cases (19.6\%) in the right lower lobe, and 6 cases (11.8\%) in the right upper lobe. High expression of PD-L1 was found in 23 cases (45.1\%), low expression of PD-L1 was found in 4 cases (7.8\%), moderate expression of PD-L1 was found in 15 cases (29.4\%). There was no significant difference between the two groups at baseline $(\mathrm{P}>0.05)$ (Table1). 
Table 1

Baseline characteristics

\begin{tabular}{|c|c|c|c|c|}
\hline & $\begin{array}{l}\text { All patients } \\
(n=51)\end{array}$ & $\begin{array}{l}\text { ICl group } \\
(n=17)\end{array}$ & $\begin{array}{l}\text { CT group } \\
(n=34)\end{array}$ & $P$ \\
\hline Age, years Median(IQR) & $54(46.5-57)$ & $52(46-57)$ & $54(47-57)$ & 0.292 \\
\hline Sex & & & & 0.164 \\
\hline Female & $28(54.9 \%)$ & $7(41.2 \%)$ & $21(61.8 \%)$ & \\
\hline Male & $23(45.1 \%)$ & $10(58.8 \%)$ & $13(38.2 \%)$ & \\
\hline ECOG performance status & & & & 0.417 \\
\hline 0 & $31(60.8 \%)$ & $9(52.9 \%)$ & $22(64.7 \%)$ & \\
\hline 1 & $20(39.2 \%)$ & $8(47.1 \%)$ & $12(35.3 \%)$ & \\
\hline Smokers & $17(33.3 \%)$ & $5(29.4 \%)$ & $12(35.3 \%)$ & 0.674 \\
\hline Family history & $8(15.7 \%)$ & $2(11.8 \%)$ & $6(17.6 \%)$ & 0.892 \\
\hline Tumor size & & & & 0.834 \\
\hline$\geq 4 \mathrm{~cm}$ & $34(66.7 \%)$ & $11(64.7 \%)$ & $23(67.6 \%)$ & \\
\hline$<4 \mathrm{~cm}$ & 17(33.3\%) & $6(35.3 \%)$ & $11(32.4 \%)$ & \\
\hline Metastases & & & & 0.36 \\
\hline single organ & $20(39.2 \%)$ & $9(52.9 \%)$ & $11(32.4 \%)$ & \\
\hline multiple organ & $31(60.8 \%)$ & $8(47.1 \%)$ & $23(67.6 \%)$ & \\
\hline Tumor location & & & & 0.516 \\
\hline left upper lobe of lung & $4(7.8 \%)$ & $2(11.8 \%)$ & $2(5.9 \%)$ & \\
\hline left lower lobe of lung & 10(19.6\%) & $2(11.8 \%)$ & $8(23.5 \%)$ & \\
\hline right upper lobe of lung & $6(11.8 \%)$ & $3(17.6 \%)$ & $3(8.8 \%)$ & \\
\hline right middle lobe of lung & 17(33.3\%) & $4(23.5 \%)$ & $13(38.2 \%)$ & \\
\hline right lower lobe of lung & $14(27.5 \%)$ & $6(35.3 \%)$ & $8(23.5 \%)$ & \\
\hline Tumor proportion score(TPS) & & & & 0.769 \\
\hline$<1 \%$ & $4(7.8 \%)$ & $2(11.8 \%)$ & $2(5.9 \%)$ & \\
\hline $1-49 \%$ & $15(29.4 \%)$ & $5(29.4 \%)$ & $10(29.4 \%)$ & \\
\hline$\geq 50 \%$ & $23(45.1 \%)$ & $8(47.1 \%)$ & $15(44.1 \%)$ & \\
\hline NA & $9(17.6 \%)$ & $2(11.8 \%)$ & $7(20.6 \%)$ & \\
\hline
\end{tabular}

The minimum follow-up time was about 5 months. The median follow-up time was 16.6 months in the $\mathrm{ICl}$ group $(95 \% \mathrm{Cl} 13.9-19.4)$ and 12.6 months in the CT group $(95 \% \mathrm{Cl}, 11.6-13.5)$. At the end of the follow-up period, 5 patients $(29.4 \%)$ in the ICl group and 3 patients (8.8\%) in the CT group continued treatment. There were no CR cases in ICI group and CT group. In ICl group, 11 patients (64.7\%) achieved PR, and 6 patients (35.3\%) achieved SD. In the CT group, 12 patients (35.3\%) achieved PR, 20 patients (58.8\%) achieved SD, and 2 patients (5.9\%) did not respond to treatment. The ORR of ICl group and CT group were 11(64.7\%) and 12(35.3\%), respectively, P=0.047; DCR was 17(100\%) and 32(94.1\%), $\mathrm{P}=0.547$ (Table2)(figure2). 
Table 2

Efficacy of $\mathrm{ICl}$ and chemotherapy treatments

\begin{tabular}{|lll|}
\hline & ICl group(n=17) & CT group(n=34) \\
\hline Best overall response & & 0 \\
\hline Complete response & 0 & $12(35.3 \%)$ \\
\hline Partial response & $11(64.7 \%)$ & $20(58.8 \%)$ \\
\hline Stable disease & $6(35.3 \%)$ & $2(5.9 \%)$ \\
\hline Progressive disease & 0 & $12(35.3 \%, 18.4-52.2)$ \\
\hline Objective response & $11(64.7 \%, 39.4-90.0)$ & $32(94.1 \%, 85.8-102.5)$ \\
\hline $\begin{array}{l}\text { Disease control } \\
\text { Data are } n \text { (\%). Confirmed complete and partial responses were assessed by the investigator according to the Response Evaluation }\end{array}$ \\
\hline
\end{tabular}

Median progression-free survival was 14.9 months $(95 \% \mathrm{Cl}, 13.2$ to 16.6$)$ in the immunotherapy group and 6.4 months (95\% $\mathrm{Cl}, 5.8$ to 7.0$)$ in the chemotherapy group [HR, $0.29 ; 95 \% \mathrm{Cl}, 0.15$ to $0.55 ; \mathrm{P}=0.003$ ]. The median overall survival was not yet achieved in the ICl group, while the median progression-free survival was 13.0 months $(95 \% \mathrm{Cl} 11.9-14.0)$ in the CT group. A total of 42 patients (82.3\%) had quantifiable PD-L1 expression. The positive rate of PD-L1 was balanced between the two treatment groups. For patients with high pD-L1 expression, median progression-free survival was 15 months $(95 \% \mathrm{Cl}, 12.8-17.2)$ in the $\mathrm{ICl}$ group and 5.0 months $(95 \% \mathrm{Cl}, 2.6-7.4)$ in the $\mathrm{CT}$ group [HR, $0.24 ; 95 \% \mathrm{Cl}, 0.09-0.62 ; \mathrm{P}<0.05]$. Among patients with $\mathrm{PD}-\mathrm{L} 1$ underexpression, median progression-free survival was 14.9 months (95\% $\mathrm{Cl}$, 0.53-29.3) in the ICl group and 3.8 months $(95 \% \mathrm{Cl}, 0-8.5)$ in the CT group [HR, 0.29; $95 \% \mathrm{Cl}, 0.09-0.98 ; \mathrm{P}=0.032]$ (figure3).

Treatment-related adverse events (both hematological and non-hematological poisoning events) occurred more frequently in the ICI group than in the CT group. In the ICI group, there were 8 (47.1\%) grade 3 and 5 (29.4\%) grade 4 treatment-related adverse reactions. In the CT group, there were 9 (26.5\%) grade 3 and 8 (23.5\%) grade 4 treatment-related adverse reactions. The most common adverse reactions in ICI group were decreased appetite 4(23.5\%), anemia 4(23.5\%), leukopenia 7(41.2\%), neutropenia $7(41.2 \%)$, lymphocyte count $4(23.5 \%)$, platelet count 4(23.5\%), and rash 4(23.5\%). Anemia 8(23.5\%), leukopenia 8(23.5\%), neutropenia 6(17.6\%), and thrombocytopenia 7(20.6\%) were most common in the CT group. Two treatment-related grade 3 selective adverse events were reported in the ICI group, of which one was hyperthyroidism and one was tracheal fistula (Table3). 
Table 3

Treatment-related adverse events

\begin{tabular}{|c|c|c|c|c|c|c|c|c|c|c|}
\hline & \multicolumn{5}{|c|}{$\mathrm{ICl}$ group $(n=17)$} & \multicolumn{5}{|c|}{ CT group $(n=34)$} \\
\hline & $\begin{array}{l}\text { Grade1- } \\
2\end{array}$ & Grade3 & Grade4 & Grade5 & ALL & $\begin{array}{l}\text { Grade1- } \\
2\end{array}$ & Grade3 & Grade4 & Grade5 & ALL \\
\hline Fatigue & $3(17.6 \%)$ & 0 & 0 & 0 & $3(17.6 \%)$ & $1(2.9 \%)$ & 0 & 0 & 0 & $1(2.9 \%)$ \\
\hline Nausea & $2(11.8 \%)$ & 0 & 0 & 0 & $2(11.8 \%)$ & $4(11.8 \%)$ & 0 & 0 & 0 & $4(11.8 \%)$ \\
\hline Vomiting & $2(11.8 \%)$ & 0 & 0 & 0 & $2(11.8 \%)$ & $4(11.8 \%)$ & 0 & 0 & 0 & $4(11.8 \%)$ \\
\hline Anorexia & $4(23.5 \%)$ & 0 & 0 & 0 & $4(23.5 \%)$ & $4(11.8 \%)$ & 0 & 0 & 0 & $4(11.8 \%)$ \\
\hline $\begin{array}{l}\text { Peripheral } \\
\text { sensory } \\
\text { neuropathy }\end{array}$ & $2(11.8 \%)$ & 0 & 0 & 0 & $2(11.8 \%)$ & $2(5.9 \%)$ & 0 & 0 & 0 & $2(5.9 \%)$ \\
\hline Hypothyroidism & $1(5.9 \%)$ & $1(5.9 \%)$ & 0 & 0 & $2(11.8 \%)$ & 0 & 0 & 0 & 0 & 0 \\
\hline Anemia & $4(23.5 \%)$ & 0 & 0 & 0 & $4(23.5 \%)$ & $8(23.5 \%)$ & 0 & 0 & 0 & $8(23.5 \%)$ \\
\hline $\begin{array}{l}\text { White blood cell } \\
\text { decreased }\end{array}$ & $5(29.4 \%)$ & $2(11.8 \%)$ & 0 & 0 & $7(41.2 \%)$ & $4(11.8 \%)$ & $1(2.9 \%)$ & $3(8.8 \%)$ & 0 & $8(23.5 \%)$ \\
\hline $\begin{array}{l}\text { Neutrophil } \\
\text { count } \\
\text { decreased }\end{array}$ & $1(5.9 \%)$ & $2(11.8 \%)$ & $4(23.5 \%)$ & 0 & $7(41.2 \%)$ & $1(2.9 \%)$ & $1(2.9 \%)$ & $5(14.7 \%)$ & 0 & $6(17.6 \%)$ \\
\hline $\begin{array}{l}\text { Lymphocyte } \\
\text { count } \\
\text { decreased }\end{array}$ & $2(11.8 \%)$ & $1(5.9 \%)$ & $1(5.9 \%)$ & 0 & $4(23.5 \%)$ & $1(2.9 \%)$ & $1(2.9 \%)$ & 0 & 0 & $2(5.9 \%)$ \\
\hline $\begin{array}{l}\text { platelet count } \\
\text { decreased }\end{array}$ & $3(17.6 \%)$ & $1(5.9 \%)$ & 0 & 0 & $4(23.5 \%)$ & $3(8.8 \%)$ & $4(11.8 \%)$ & 0 & 0 & $7(20.6 \%)$ \\
\hline Hyponatremia & $2(11.8 \%)$ & 0 & 0 & 0 & $2(11.8 \%)$ & $1(2.9 \%)$ & 0 & 0 & 0 & $1(2.9 \%)$ \\
\hline Hypokalemia & $2(11.8 \%)$ & 0 & 0 & 0 & $2(11.8 \%)$ & $1(2.9 \%)$ & $1(2.9 \%)$ & 0 & 0 & $2(5.9 \%)$ \\
\hline ALT increased & $1(5.9 \%)$ & 0 & 0 & 0 & $1(5.9 \%)$ & 0 & $1(2.9 \%)$ & 0 & 0 & $1(2.9 \%)$ \\
\hline AST increased & $2(11.8 \%)$ & 0 & 0 & 0 & $2(11.8 \%)$ & $1(2.9 \%)$ & 0 & 0 & 0 & $1(2.9 \%)$ \\
\hline $\begin{array}{l}\text { Blood bilirubin } \\
\text { increased }\end{array}$ & $1(5.9 \%)$ & 0 & 0 & 0 & $1(5.9 \%)$ & $1(2.9 \%)$ & 0 & 0 & 0 & $1(2.9 \%)$ \\
\hline $\begin{array}{l}\text { Rash maculo- } \\
\text { papular }\end{array}$ & $4(23.5 \%)$ & 0 & 0 & 0 & $4(23.5 \%)$ & $2(5.9 \%)$ & 0 & 0 & 0 & $2(5.9 \%)$ \\
\hline Tracheal fistula & 0 & $1(5.9 \%)$ & 0 & 0 & $1(5.9 \%)$ & 0 & 0 & 0 & 0 & 0 \\
\hline Overall & 41 & 8 & 5 & $0 \quad 54$ & & 38 & 9 & 8 & 0 & 55 \\
\hline
\end{tabular}

FBXW7 mutations were negatively correlated with the efficacy of immunotherapy. TP53 mutation, MRE11A p.V198S mutation and PTEN p.T319FS mutation were positively correlated with the efficacy of immunotherapy. MYC amplification, NRAS P.q61L mutation and FGFR3TACC3(F17:T10) fusion were negatively correlated with chemotherapy efficacy. APC p.Q1378* mutation and SMAD4 p.K110Ter mutation were positively correlated with chemotherapy efficacy (Table4)(figure4). 
Table 4

Genic mutation and related clinical stated

\begin{tabular}{|c|c|c|c|c|}
\hline ICl patients genic mutation & $\begin{array}{l}\text { PFS, } \\
\text { months }\end{array}$ & $\begin{array}{l}\text { OS, } \\
\text { months }\end{array}$ & State & Relativity of treatment \\
\hline FBXW7 mutation(0.4\%) & 3 & 5 & Death & Negative \\
\hline TP53 mutation & 13 & 19 & Alive & Positive \\
\hline MRE11A p.V198S (6.6\%) & 15.4 & 22 & Alive & Positive \\
\hline PTEN p.T319fs(2.81\%) & 16.3 & 22 & Alive & Positive \\
\hline $\begin{array}{l}\text { ATR p.E2354V (0.54\%) } \\
\text { DDR2 copynumber increase } 6 \\
\text { NTRK1 copynumber increase } 8 \\
\text { RIT1 copynumber increase } 10 \\
\text { JAK3 copynumber increase } 6\end{array}$ & 1.4 & 3 & Death & Unknown \\
\hline $\begin{array}{l}\text { EGFR p.A871V (1.3\%) } \\
\text { EGFR p. R831H (1.0\%) } \\
\text { BRAF p.G466R (1.5\%) } \\
\text { FBXW7 p.S349N (3.6\%) } \\
\text { IDH2 p.R140Q (1.1\%) } \\
\text { KIT p.D572N (2.6\%) } \\
\text { NF1 p.L1480F (3.7\%) } \\
\text { NF1 p.W2075Ter (3.6\%) } \\
\text { PTEN p.C105Y (2.3\%) } \\
\text { RET p.R912W (2.4\%) }\end{array}$ & 14.3 & 20 & Alive & Unknown \\
\hline PTEN mutation & 5.3 & 6 & Alive & Unknown \\
\hline NOTCH p.G403R (5.8\%) & 8.4 & 16 & Alive & Unknown \\
\hline CT patients genic mutation & $\begin{array}{l}\text { PFS, } \\
\text { months }\end{array}$ & $\begin{array}{l}\text { OS, } \\
\text { months }\end{array}$ & State & Relativity of treatment \\
\hline MYC copynumber increase 8 & 1.7 & 7 & Death & Negative \\
\hline NRAS p.Q61L (14.5\%) & 2.4 & 8 & Death & Negative \\
\hline FGFR3-TACC3(F17:T10) fusion & 3.6 & 9 & Death & Negative \\
\hline APC p.Q1378* (42.6\%) & 7.8 & 13 & Alive & Positive \\
\hline SMAD4 p.K110Ter (19.5\%) & 8.8 & 13.5 & Alive & Positive \\
\hline $\begin{array}{l}\text { TP53 p.R248W(17.87\% ) } \\
\text { TRIM58 p.A300V(21.13\%) }\end{array}$ & 7.9 & 15 & Alive & Unknown \\
\hline $\begin{array}{l}\text { PIK3CA p.Q546K (23.36\%) } \\
\text { TP53 p.R249Q (0.17\%) }\end{array}$ & 7.3 & 14 & Death & Unknown \\
\hline
\end{tabular}




\begin{tabular}{|lllll|}
\hline ICI patients genic mutation & $\begin{array}{l}\text { PFS, } \\
\text { months }\end{array}$ & $\begin{array}{l}\text { OS, } \\
\text { months }\end{array}$ & State & Relativity of treatment \\
\hline $\begin{array}{l}\text { RET-CCDC6 fusion } \\
\text { TP53 p.R273H (38.2\%) }\end{array}$ & 3.5 & 11 & Alive & Unknown \\
\hline Positive and negative genes associated with treatment of PPLELC. & \\
\hline
\end{tabular}

\section{Discussion}

PPLELC is a virus-associated tumor. In other virus-associated tumors, such as nasopharyngeal cancer and cervical cancer, immune checkpoint blockers have shown good efficacy, but the effect of immune checkpoint inhibitors on PPELC is still unknown. The prognosis of metastatic PPLELC is poor, so it is worth exploring the effect of immunocheckpoint inhibitors on PPLELC. At present, immune checkpoint inhibitors have been reported for PPLELC, but most of them are case reports or single-center cohort studies.

In 2016, Chul Kim et al. first reported a patient with PPLELC who received nivolumab after postoperative recurrence ${ }^{14} \square$ Cases of immunotherapy from multiple centers were subsequently reported, with first-line treatment of PFS up to 25 and 27 months ${ }^{25}$. Immunotherapy also achieved good results for both posterior and translinear therapy, and Na Zhou et al. reported that patients with PPLELC who received pembrolizumab at 4 lines remained stable for 12 months ${ }^{27}, 28$. The included objects in this retrospective study are mainly six large third-class a hospitals in China, which are more representative.

Immunotherapy improves survival and quality of life in patients with metastases or relapses, and its predictive biomarkers are microsatellite instability-high(MSI-H)/mismatch repair defects and programmed death-1 (PD-1)/PD-L1 protein expression. The frequency of PD-L1 positivity in PPLELC tumors is high. When TPSs $\geq 1 \%, 5 \%, 10 \%$ and $50 \%$, the positive rates were $96.6 \%, 91.5 \%, 83.1 \%$ and $61.0 \%$, respectively ${ }^{17}$. Meta-analysis suggested that the incidence of programmed cell death ligand 1(PD-L1) expression in PPLELC ranged from $63.3-75.8 \%{ }^{30}$. Some studies have found that MSI-H exists in $4 \%(2 / 57)$ of PPLELC. The existence of mismatch repair defect phenotype and high prevalence of PD-L1 in PPLELC may provide evidence for immunotherapy.

$\mathrm{ICl}$ combined with chemotherapy showed significantly better efficacy for metastatic PPLELC than chemotherapy alone, and the level of PDL1 expression indicated the benefit of efficacy. The possible reasons are as follows: for most patients with PPLELC, the pathology shows a large amount of lymphocyte infiltration, and studies have reported that both active and suppressed tumor infiltrating lymphocytes contribute to better results in PPLELC immunotherapy ${ }^{31}$. For other predictive biomarkers, it has been suggested that EBV infection may be a biomarker of favorable outcomes in immunotherapy ${ }^{32-35}$.

The mutant spectrum between PPLELC and NPC has a higher similarity ${ }^{30}$, However, the mutation status of the classic oncodriver and suppressor genes in lung cancer is relatively low in lung lymphoepithelioid carcinoma ${ }^{13,36,37}$. EGFR and ALK mutation rates are reported in $30 \%-40 \%$ and $6 \%-8 \%$ of NSCLC patients, respectively. Only $17.4 \%$ (8/46) of PPLELC patients had EGFR mutations, and most (7/8) were not classic EGFR mutations ${ }^{38}$.

As for immunotherapy prediction genes, this study reported gene changes that may be related to immunotherapy in PPLELC, including FBXW7 mutation in negative related genes, TP53 mutation, MRE11A mutation and PTEN mutation in positive related genes. This is contrary to the correlation between FBXW7 and PTEN and the efficacy of immune checkpoint inhibitors reported in the previous literature ${ }^{39,40}$. Cause the cancer species is different, the mutation site is different, and the number of cases in this study is relatively small. Therefore, individual differences are more prominent.

\section{Conclusions}

Immunocheckpoint inhibitors are clinically beneficial and sufficiently safe compared to current therapies for patients with metastatic PPLELC to warrant further expansion of the patient population or extended follow-up to further observe survival benefits. FBXW7 mutation were negatively and TP53 mutation, MRE11A p.V198S mutation, PTEN p.T319FS mutation were positively correlated with the efficacy of immunotherapy.

\section{Abbreviations}

PPLELC: Primary pulmonary lymphoepithelioma-like carcinoma 
NSCLC: non-small cell lung cancer

ICls: immune checkpoint inhibitors

CT: chemotherapy

HR: Hazard Ratio

Cl: confidence interval

NPC: nasopharyngeal carcinoma

WHO: world Health Organization

EBV: epstein-Barr virus

EBER: EBV-encoded RNA

ECOG: Eastern Cooperative Oncology Group

RECIST: Response Assessment Criteria

TNM: tumor-node-metastasis

PD-1: programmed death-1

PD-L1: programmed cell death ligand 1

MSI-H: microsatellite instability-high

\section{Declarations}

\section{Authors' contributions}

HJB: Conceptualization; Data curation; Resources; Writing-original draft; Writing-review \& editing.

LZM, LXL: Methodology; Visualization; Writing-review \& editing.

BSZ, JZ, GBP, XTL: Resources; Validation; Visualization.

YHF, HHB: Formal analysis; Methodology; Software; Writing-original draft.

SDM: Funding acquisition; Project administration; Supervision; Visualization.

\section{Acknowledgements}

The authors are grateful to all staff at the study centre who contributed to this study.

\section{Competing interests}

The authors declare that they have no competing interests.

\section{Availability of data and materials}

All data generated or analysed during this study are included in this published article.

\section{Consent for publication}

Not Applicable.

\section{Ethics approval and consent to participate}

This study was approved by the Ethics Review Board of Nanfang Hospital, Southern Medical University (Guangzhou, China). 


\section{Funding}

This study was supported by the Antitumor Angiogenesis Targeted Therapy Research Fund of the Chinese Society of Clinical Oncology (YS2016-004), the Nanfang Hospital Dean's Fund (2018B013), and the Wu Jieping Medical Foundation (320.6750.19061)

\section{References}

1. Ambrosio MR, Rocca BJ, Onorati M, et al. Lymphoepithelioma-Like Carcinoma of the Ovary. Int J Surg Pathol. 2011;19(4):514-517.

2. Anand A, Zayac A, Curtiss C, Graziano S. Pulmonary Lymphoepithelioma-like Carcinoma Disguised as Squamous Cell Carcinoma. $J$ Thorac Oncol. 2018;13(5):E75-E76.

3. Hayashi T, Haba R, Tanizawa J, et al. Cytopathologic Features and Differential Diagnostic Considerations of Primary Lymphoepithelioma-Like Carcinoma of the Lung. Diagn Cytopathol. 2012;40(9):820-825.

4. Xie Z, Liu L, Lin X, et al. A multicenter analysis of genomic profiles and PD-L1 expression of primary lymphoepithelioma-like carcinoma of the lung. Modern Pathol. 2020;33(4):626-638.

5. Travis WD, Brambilla E, Müller-Hermelink HK, Harris CC. Pathology and genetics of tumours of the lung, pleura, thymus and heart. 2004.

6. Available N. Pathology and Genetics of Tumours of the Lung, Pleura, Thymus and Heart (IARC/World Health Organization Classification of Tumours). Intl Agency for Research on Cancer. 2015.

7. Begin LR, Eskandari J, Joncas J, Panasci L. Epstein-Barr virus related lymphoepithelioma-like carcinoma of lung. J Surg Oncol. 1987;36(4):280-283.

8. Han AJ, Xiong M, Zong YS. Association of Epstein-Barr virus with lymphoepithelioma-like carcinoma of the lung in southern China. Am J Clin Pathol. 2000;114(2):220-226.

9. Jiang W, Wang R, Pan X, et al. Clinicopathological features and prognosis of primary pulmonary lymphoepithelioma-like carcinoma. J Thorac Dis. 2016;8(9):2610-2616.

10. Jiang W, Wang R, Pan X, et al. Clinicopathological features and prognosis of primary pulmonary lymphoepithelioma-like carcinoma. $J$ Thorac Dis. 2016;8(9):2610-2616.

11. Hu Y, Ren S, Liu Y, Han W, Liu W. Pulmonary Lymphoepithelioma-Like Carcinoma: A Mini-Review. Oncotargets Ther. 2020;13:3921-3929.

12. Castro CY, Ostrowski ML, Barrios R, et al. Relationship between Epstein-Barr virus and lymphoepithelioma-like carcinoma of the lung: a clinicopathologic study of 6 cases and review of the literature. Hum Pathol. 2001;32(8):863-872.

13. Yeh Y, Kao H, Lee K, Wu M, Ho H, Chou T. Epstein-Barr Virus-Associated Pulmonary Carcinoma Proposing an Alternative Term and Expanding the Histologic Spectrum of Lymphoepithelioma-like Carcinoma of the Lung. Am J Surg Pathol. 2019;43(2):211-219.

14. Kim C, Rajan A, Debrito PA, Giaccone G. Metastatic lymphoepithelioma-like carcinoma of the lung treated with nivolumab: a case report and focused review of literature. Translational lung cancer research. 2016-01-01 2016;5(6):720-726.

15. Darrason M, Martin A, Soussan M, et al. Immunotherapy for LELC: Case Report and a Focused Review. Clin Lung Cancer. 2019;20(3):e393-e401.

16. Hong S, Liu D, Luo S, et al. The genomic landscape of Epstein-Barr virus-associated pulmonary lymphoepithelioma-like carcinoma. Nat Commun. 2019;10(3108).

17. Wu Q, Wang W, Zhou P, et al. Primary pulmonary lymphoepithelioma-like carcinoma is characterized by high PD-L1 expression, but low tumor mutation burden. Pathology - Research and Practice. 2020;216(8):153043.

18. Chen J, Gu C, Chen X, et al. Clinicopathological and prognostic analyses of 86 resected pulmonary lymphoepithelioma-like carcinomas. J Surg Oncol. 2020.

19. Lin Z, Situ D, Chang X, et al. Surgical treatment for primary pulmonary lymphoepithelioma-like carcinoma. Interact Cardiov Th. 2016;23(1):41-46.

20. Zhu N, Lin S, Xu N, Chen L, Piao Z, Cao C. Primary pulmonary lymphoepithelioma-like carcinoma accompanied by hypertrophic pulmonary osteoarthropathy in a non-epidemic region: a case report and literature review. $J$ Int Med Res. 2020;48(030006052096581611).

21. Lee K, Wu M, Jhang Y, Chen C, Yen Y, Chen Y. Computed tomography-based differentiation of primary pulmonary lymphoepitheliomalike carcinoma and small-cell lung cancer. J Chin Med Assoc. 2020;83(10):936-942.

22. He J, Shen J, Pan H, Huang J, Liang W, He J. Pulmonary lymphoepithelioma-like carcinoma: a Surveillance, Epidemiology, and End Results database analysis. J Thorac Dis. 2015;7(12):2330-2338.

23. Liang Y, Wang L, Zhu Y, et al. Primary pulmonary lymphoepithelioma-like carcinoma. Cancer-Am Cancer Soc. 2012;118(19):4748-4758.

Page $11 / 16$ 
24. Ho JC, Wong MP, Lam WK. Lymphoepithelioma-like carcinoma of the lung. Respirology. 2006;11(5):539-545.

25. Kumar V, Dave V, Harris J, Huang Y. Response of advanced stage recurrent lymphoepithelioma-like carcinoma to nivolumab. Immunotherapy-Uk. 2017;9(12):955-961.

26. Narayanan A, Knollmann FD, Walby JAS, Lim S, Gandara DR, Riess JW. EBV-positive Primary Pulmonary Lymphoepithelioma-like Carcinoma Response to PD-L1 Blockade. Clin Lung Cancer. 2019;20(3):e238-e241.

27. Zhou N, Lin Y, Peng X, Wang Y, Wang Y. Thorough survey and analysis of pulmonary lymphoepithelioma-like carcinoma in Macau and multimodality treatment for advanced disease. Lung Cancer. 2019;138:116-123.

28. Tang Z, Fang R, Tong G, Liu P, Ou ZA, Tang Y. Overcoming resistance to anti-PD-1 immunotherapy in lymphoepithelioma-like carcinoma: A case report and review of the literature. Lung Cancer. 2020;146:335-340.

29. Qiu Z, Zhou P, Wang K. Primary Pulmonary Lymphoepithelioma-Like Carcinoma Response Favorably To Nivolumab: A Case Report. Oncotargets Ther. 2019;12:8595-8600.

30. Chau S, Tong JH, Chow C, et al. Distinct Molecular Landscape of Epstein-Barr Virus Associated Pulmonary Lymphoepithelioma-Like Carcinoma Revealed by Genomic Sequencing. Cancers. 2020;12(20658).

31. Kasai K, Kon S, Sato N, et al. Case report of lymphoepithelioma-like carcinoma of the lung-lymphoid population consisting of cytotoxic T cells in resting state. Pathol Res Pract. 1999-01-19 1999;195(11):773-779.

32. Xie M, Wu X, Wang F, et al. Clinical Significance of Plasma Epstein-Barr Virus DNA in Pulmonary Lymphoepithelioma-like Carcinoma (LELC) Patients. J Thorac Oncol. 2018-02-01 2018;13(2):218-227.

33. Hong S, Liu D, Luo S, et al. The genomic landscape of Epstein-Barr virus-associated pulmonary lymphoepithelioma-like carcinoma. Nat Commun. 2019-07-16 2019;10(1):3108.

34. Kim SJ, Hyeon J, Cho I, Ko YH, Kim WS. Comparison of Efficacy of Pembrolizumab between Epstein-Barr VirusPositive and Negative Relapsed or Refractory Non-Hodgkin Lymphomas. Cancer Res Treat. 2019-04-01 2019;51(2):611-622.

35. Kang HJ, Oh JH, Chun SM, et al. Immunogenomic landscape of hepatocellular carcinoma with immune cell stroma and EBV-positive tumor-infiltrating lymphocytes. J Hepatol. 2019-07-01 2019;71(1):91-103.

36. Chang Y, Wu C, Shih J, Lee Y. Unique p53 and epidermal growth factor receptor gene mutation status in 46 pulmonary lymphoepithelioma-like carcinomas. Cancer Sci. 2011;102(1):282-287.

37. Liu Q, Ma G, Yang H, et al. Lack of epidermal growth factor receptor gene mutations in exons 19 and 21 in primary lymphoepitheliomalike carcinoma of the lung. Thorac Cancer. 2014;5(1):63-67.

38. Yin K, Feng HB, Li LL, et al. Low frequency of mutation of epidermal growth factor receptor (EGFR) and arrangement of anaplastic lymphoma kinase (ALK) in primary pulmonary lymphoepithelioma-like carcinoma. Thorac Cancer. 2020-02-01 2020;11(2):346-352.

39. Peng W, Chen JQ, Liu C, et al. Loss of PTEN Promotes Resistance to T Cell-Mediated Immunotherapy. Cancer Discov. 2016-02-01 2016;6(2):202-216.

40. Kato S, Goodman A, Walavalkar V, Barkauskas DA, Sharabi A, Kurzrock R. Hyperprogressors after Immunotherapy: Analysis of Genomic Alterations Associated with Accelerated Growth Rate. Clin Cancer Res. 2017-08-01 2017;23(15):4242-4250.

\section{Figures}




\section{8 patients were diagnosed with}

metastatic primary pulmonary lymphoepithelioma-like carcinoma

107 patients were diagnosed with metastatic primary pulmonary lymphoepithelioma-like carcinoma

61 were excluded for previously treated

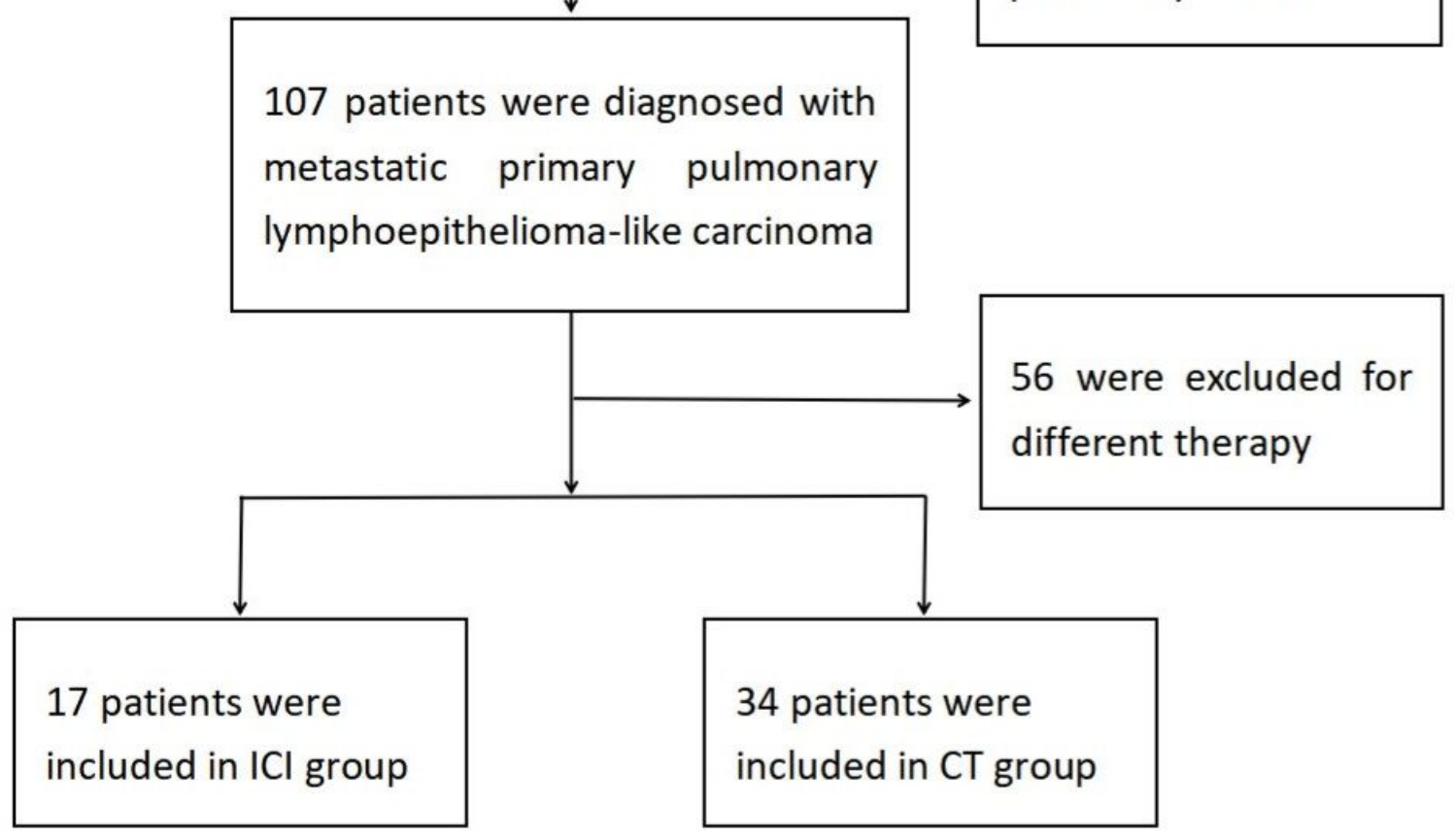

Figure 1

Study enrolment. A total of 168 patients diagnosed with metastatic PPLELC from six grade A patients from August 2018 to August 2020 were selected, and 51 patients were eventually included. 61 patients were excluded because of non-first-line treatment. 56 patients were excluded because the treatment regimen did not meet the requirements of the study. Finally, of 51 patients, 17 were included in ICl group combined with first-line immunotherapy, and 34 patients were included in CT group with first-line chemotherapy. 
a

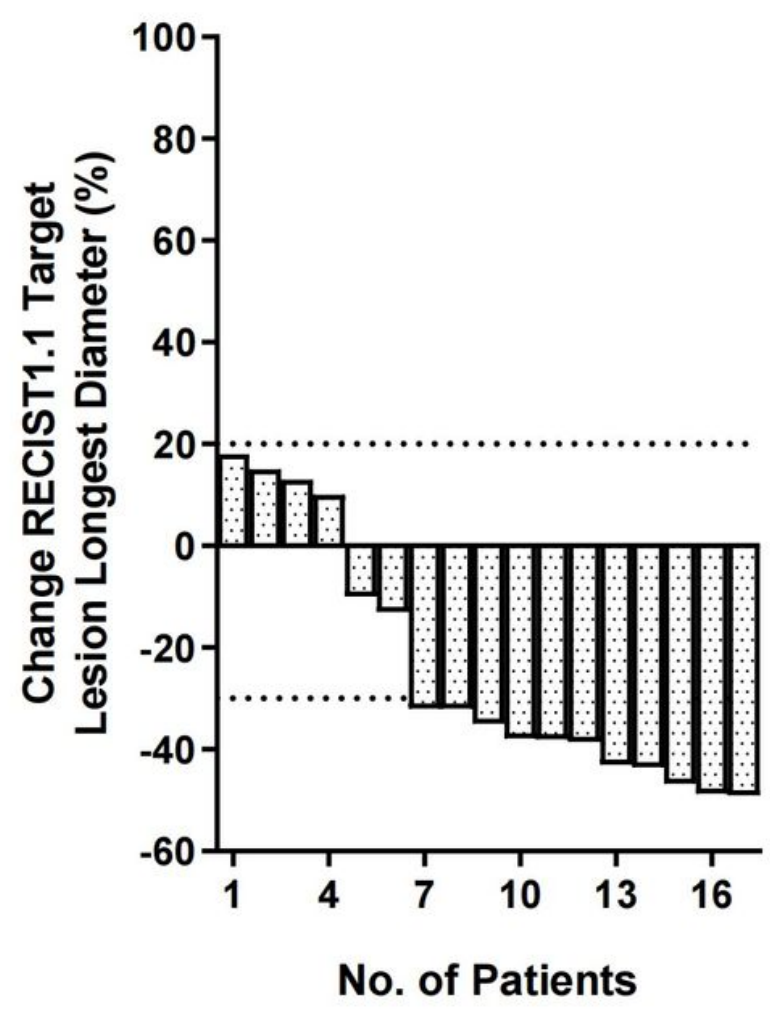

b

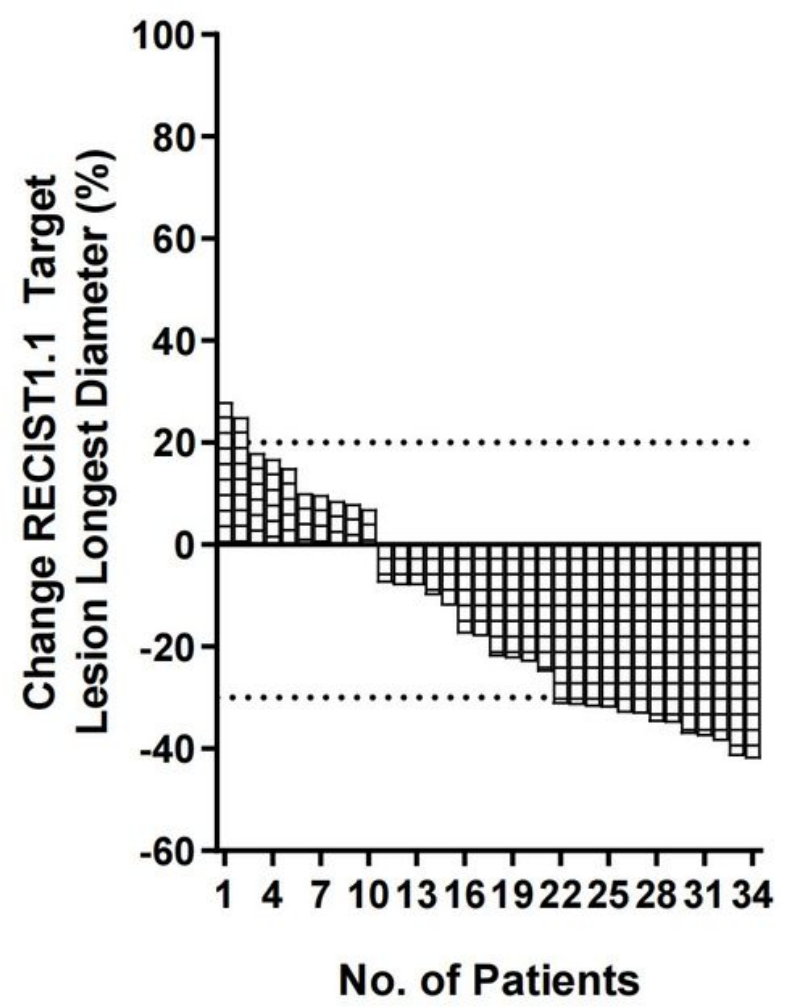

Figure 2

Waterfall diagram of optimal efficacy evaluation in ICl group and CT group. A) In ICl group, 6 patients achieved SD status and 11 patients achieved PR status. B) In the CT group, 12 patients achieved SD status and 20 patients achieved PR status. 
A All patients

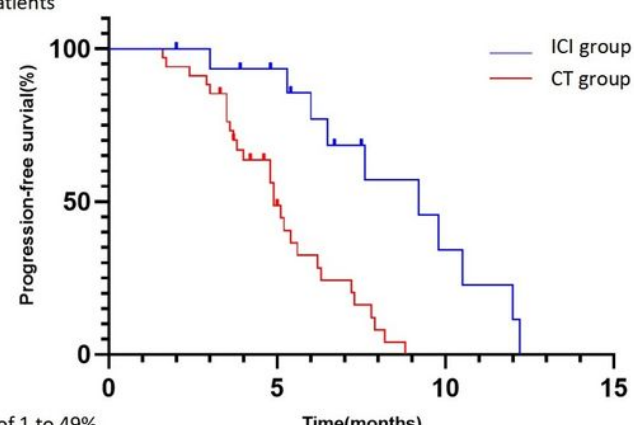

B TPS of 1 to $49 \%$

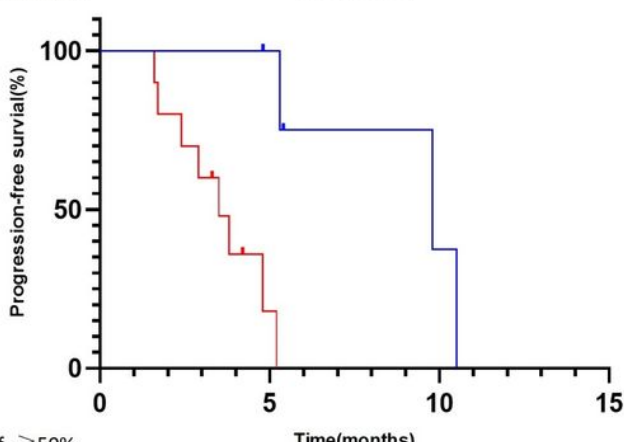

C TPS of $\geqslant 50 \%$

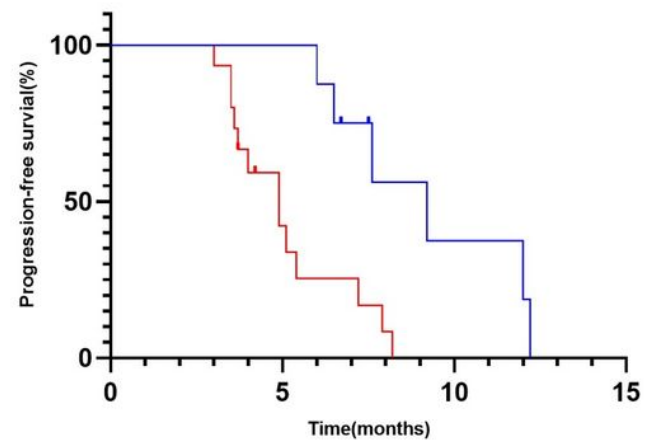

\section{Figure 3}

PFS in ICl group and CT group. A) The median PFS was 14.9 months in the $\mathrm{ICl}$ group and 6.4 months in the CT group [HR, 0.29 ; $95 \% \mathrm{Cl}, 0.15$ $0.55 ; \mathrm{P}<0.05]$. B) Among patients with $\mathrm{PD}-\mathrm{L} 1$ underexpression, median progression-free survival was 14.9 months $(95 \% \mathrm{Cl}, 0.53-29.3)$ in the $\mathrm{ICl}$ group and 3.8 months $(95 \% \mathrm{Cl}, 0-8.5)$ in the CT group [HR, 0.29; 95\% Cl, 0.09-0.98; P=0.032]. C) For cancer patients with high PD-L1 expression, median progression-free survival was 15 months $(95 \% \mathrm{Cl}, 12.8-17.2)$ in the $\mathrm{ICl}$ group and $5.0 \mathrm{months}(95 \% \mathrm{Cl}, 2.6-7.4)$ in the $\mathrm{CT}$ group [HR, 0.24; 95\% Cl, 0.09-0.62; $\mathrm{P}<0.05]$. 
CT group

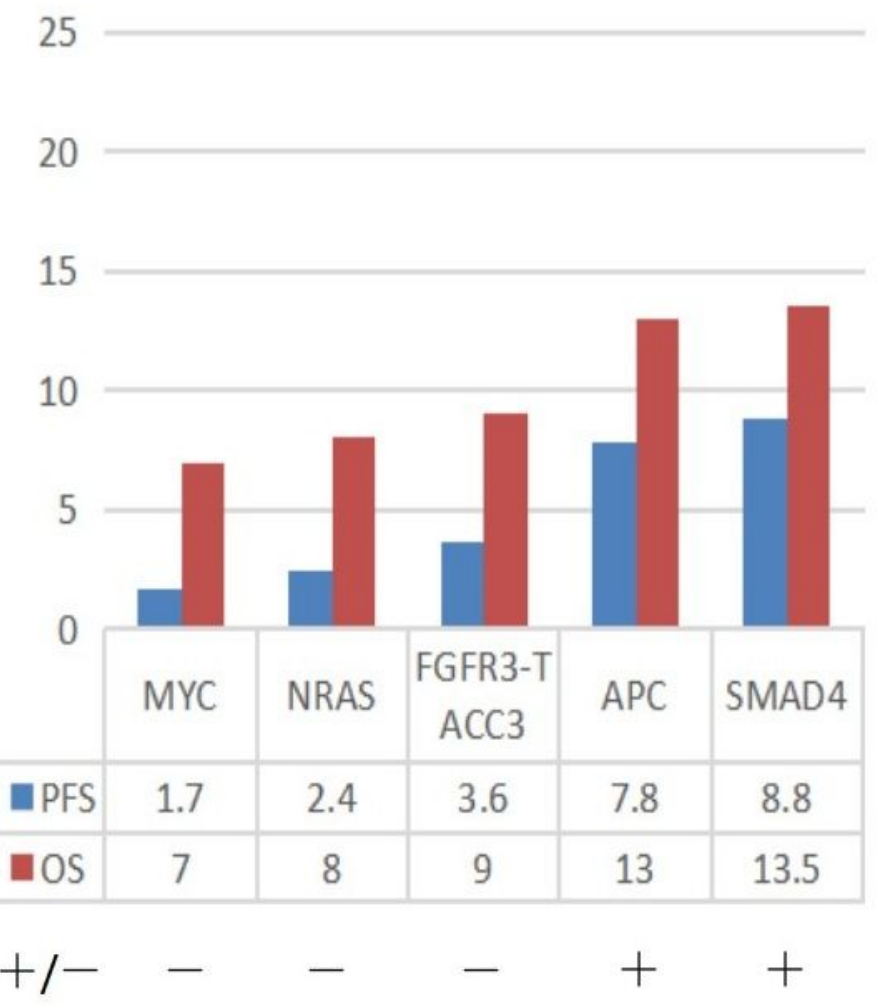

$\mathrm{ICl}$ group

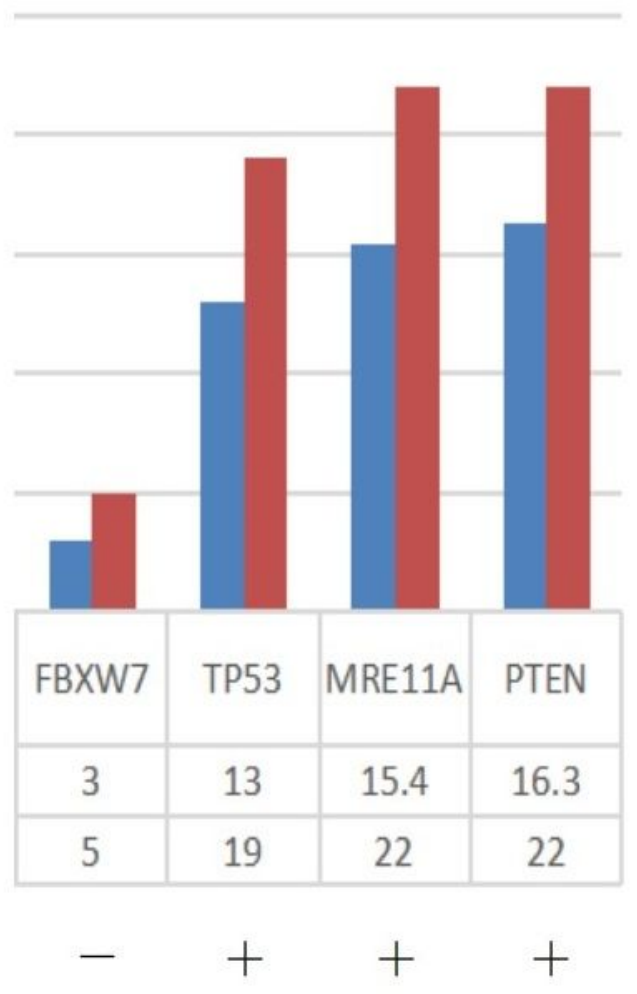

\section{Figure 4}

Effect prediction genes in ICl group and CT group. FBXW7 mutation was negatively correlated with immunotherapy efficacy. TP53 mutation, MRE11A p.V198S mutation and PTEN p.T319fs mutation were positively correlated with the efficacy of immunotherapy. MYC amplification, NRAS p.Q61L mutation and FGFR3-TACC3(F17:T10) fusion were negatively correlated with chemotherapy efficacy. APC p.Q1378* mutation and SMAD4 p.K110Ter mutation were positively correlated with chemotherapy efficacy. 